\title{
PROTOCOLO PARA CIRURGIAS EM CALVÁRIA DE RATOS PARA SER UTILIZADO NO BIOTÉRIO DA UNIVERSIDADE ESTADUAL DE FEIRA DE SANTANA
}

\author{
Leila Maria Ferreira Ribeiro'; Dario Augusto Oliveira Miranda ${ }^{2}$; Antonio Cesar \\ Azevedo $^{3}$; Liliane Brito de Oliveira ${ }^{4}$; Rafael Cabral de Albuquerque Souza ${ }^{4}$
}

1.Bolsista FAPESB, Graduando em odontologia, Universidade Estadual de Feira de Santana, email:leilaribeiro.cd@hotmail.com

2.Orientador, Departamento de Saúde, Universidade Estadual de Feira de Santana, email:drdariomiranda@icloud.com

3.Pesquisador, Departamento de Saúde, Universidade Estadual de Feira de Santana, email:antoniocesarazevedo@gmail.com

4. Bolsista PROBIC, Graduando em Odontologia, Universidade Estadual de Feira de Santana, email:lilianeboliveira@outlook.com

5. Bolsista PROBIC, Graduando em Odontologia, Universidade Estadual de Feira de Santana, email: rafael_cabral27@live.com

\section{Introdução}

Palavras-chave: Reparação óssea, Calvária de ratos, Protocolo

O osso é considerado o segundo tecido mais transplantado, sendo superado apenas pelo transplante sanguíneo (BOYCE et al., 1999; VAN HEESTANDSWINTOWSKI, 1999). Embora muitas fraturas ósseas cicatrizam naturalmente sem complicações, 5 a $10 \%$ dos pacientes apresentam problemas na cicatrização como a persistência de defeitos ósseos, cicatrização prejudicada ou ambos (EINHOM 1999). A presença de defeitos ósseos na regiãomaxilofacial, decorrentes de doença inflamatória prévia, trauma, deformidades anatômicas ou congênitas constitui um dos maiores problemas terapêuticos(MARDAS et al., 2008) e uma situação desafiadora, particularmente nos casos de grandes deformidades, nas quais a capacidade regenerativa fisiológica do tecido é excedida( BURG et al.,2000). A reabsorção óssea alveolar também ocorre após a exodontia, sendo que $50 \%$ da largura do rebordo alveolar é perdida após 12 meses, e 2/3 desta perda ocorre nos primeiros 3 meses de cicatrização(SCHROPP et al., 2003), sendo as paredes do alvéolo reduzidas tanto em altura como espessura(ARAÚJO et al., 2001; ARAÚJO et al., 2005), e como alterações dimensionais mais proeminentes na parede óssea vestibular (PIETROKOVSKI\&MASSLER 1967; PIETROKOVSKI et al., 2007). Essas alterações podem interferir negativamente na instalação de implantes osseointegráveis e influenciar no sucesso do tratamento com prótese fixas ou removíveis, e assim comprometer a reabilitação estética e funcional do paciente (SCHROPPET al.,2003). O osso bovino é um enxerto xenógeno utilizado como alternativa ao enxerto autógeno (EZIRGANLIET al., 2013; 2014) por apresentar similaridade estrutural ao osso humano (HALLMAN\& THOR, 2008). Este material já possui bastante documentação de suas propriedades osteocondutivas em vários procedimentos de regeneração óssea (Baldini et al., 2011). Acelerar o processo da reabilitação oral é ainda um objetivo clínico desafiador e importante (KHADRAET al., 2004), e a busca por técnicas que beneficiem e acelerem o reparo ósseo diminuindo o tempo do tratamento tem sido amplamente pesquisadas. A utilização do laser de baixa intensidade (LB) é uma das possíveis terapias coadjuvantes neste processo. O LB possui efeitos biomodulatórios, e existem relatos de que estimula o reparo ósseo (MERLIET al., 2005; PEREIRA et al., 2009) afetando proliferação, diferenciação e adesão celular( MARLI et al., 2005).

\section{Metodologia}


Uma busca ativa de protocolos de uso da calvária de ratos em pesquisas de reparo ósseo foi realizada nas bases de dados PUBMED, Science direct, LILACS e Google Acadêmico. Para esta busca, serão empregados os descritores: protocolos reparo ósseo, calvária, rato e pesquisa experimental. Com os protocolos da literatura em mãos, será confeccionado o protocolo a ser utilizado na pesquisa. Será feito testes pilotos utilizando a calvária, fêmur, tíbia, mandíbula e intramuscular de ratos para viabilizar o protocolo. Após análise e discussão, a equipe de pesquisadores definirá o protocolo padrão a ser utilizado para esta e outras pesquisas em ratos, no biotério da UEFS.

\section{Resultados e discussão}

Os Modelos em calvária, por conseguinte, têm muitas semelhanças com a região bucomaxilofacial humana do ponto de vista embrionário, já que a mesma desenvolve-se a partir de uma origem membranosa, assim como os ossos da face. Do ponto de vista fisiológico, o osso cortical da calvária assemelha-se a uma mandíbula atrófica (SCHMITZ, HOLLINGER, 1986; SPICER et al, 2012). O modelo ideal tem que possuir as seguintes características: ser altamente reprodutível; ter versatilidade em seu uso para vários tipos de materiais e estratégicas; que possa oferecer facilidade em vários tipos de análise; que o tempo de experimentação seja o mínimo possível para que possa gerar dados estatisticamente significantes; que tenha baixa morbidade e mortalidade; e, que tenha um custo compatível com a pesquisa a ser realizada. Neste sentido a utilização de modelos com ratos tem se mostrado ideal para este tipo de pesquisa (PEARCE, et al, 2007). Para a implementação do protocolo de cirugias em ratos, no biotério da uefs, e para padronizar a pesquisa foi feita uma pesquisa nas bases de dados e o modelo que nos permimitiu seguir os protocolos desde a cirurgia até a eutanásia foi o protocolo específico internacional para avaliação de processos de reparo ósseo em defeitos críticos na calvária de ratos, publicado na Revista Nature, em 2012, que padronizou este método de pesquisa (SPICER et al, 2012). O modelo utilizado, da calvária de ratos, é adequado em termos de custo, disponibilidade do animal e facilidade da técnica. Ratos têm menor custo de obtenção e manutenção, sendo mais fáceis de abrigar do que animais maiores, conferindo um benefício econômico imediato (AYBAR ODSTRCIL; TERRITORIALE; MISSANA, 2005; SPICER et al., 2012). Os defeitos de tamanho crítico são definidos como defeitos em que o menor diâmetro intraósseo não possui a capacidade de se reparar espontaneamente, desde que não seja inserido um material que tenha capacidade osteogênica, osteoindutora e/ou osteocondutora (SCHMITZ e HOLLINGER, 1986). Não existe um consenso na literatura sobre qual seria o tamanho padrão do defeito de tamanho crítico. Para alguns autores (BOSH et al.,1995; MARDAS et al., 2011), o defeito de tamanho crítico na calvária de ratos é de $5 \mathrm{~mm}$; já outros autores, como DAHLIN et al. (1991) e MOKBEL et al. (2008), preconizam um diâmetro de 8mm. No presente estudo, foi confeccionado um defeito por meio da utilização de uma trefina de $7 \mathrm{~mm}$ de diâmetro interno, e que, ao fim da fresagem, constituía um defeito de $8 \mathrm{~mm}$, devido à espessura do metal da broca. A utilização do modelo da calvária é uma vantagem, pois é um método que este é apropriado para estudos comparativos com a área maxilofacial humana devido a algumas semelhanças fisiológicas entre elas. Anatomicamente a calvária consiste em duas folhas corticais separadas pelo osso reticulado, como a mandíbula; fisiologicamente, o padrão de cura também é semelhante à maxila. Uma desvantagem é o pequeno tamanho da calvária de rato e a delicadeza da dura-máter subjacente implicam num grande desafio técnico cirúrgico. O uso da trefina, indicada no protocolo para a realização deste trabalho, pode danificar a dura-máter, inibindo o processo de reparo. Esta é tida como de importância neste processo, já que é 
fonte primária de células osteogênicas e de fatores osteoindutores (AALAMI et al, 2004; COOPER et al, 2010).

\section{Conclusão}

Com este protocolo de cirurgias em calvárias de ratos, seguindo as condições éticas e condições do Biotério da UEFS, foi possível executar o estudo experimental do estudo caso-controle - Avaliação do Efeito do Laser de Baixa Intensidade, Associado ou não a Enxerto Ósseo de Origem Bovina, na Reparação Óssea em Calvária de Ratos: Estudo Radiográfico e Histológico", conseguindo reproduzir o métodos entre os grupos e gerar resultados com significância estatística.

\section{Referências}

AYBAR ODSTR, A, TERRITORIALE, E, MISSANA, L. An Experimental Modo in calvari to Evaluate Bone Therapias. Acta Odontológica Latinoamericana - AOL, 2005, 18 (2): 63-7. AALAMI, OO, NACAMULLI, RP, LENTON, KA, COWAN, CM, FANG, TD, FONG, KD, et al. Applications of a Mouse Model of Calvarial Healing: Differences in Regenerative Abilities of Juveniles and Adults. Plastic and reconstructive surgery. 2004 Sep 1;114(3):713-20 BOSH, C.; MELSEN, B.; VARGERVIK, K. Guided bone regeneration in calvarial bone defects using polytetrafluoroethylene membrane. Cleft Palate Craniofac J. v. 32, p. 311-317, 1995. COOPER, GM, MOONEY, MP, GOSAI,AK, CAMPBELL, PG, LOSEE, JE, HUARD, J. Testing the Critical Size in Calvarial Bone Defects: Revisiting the Concept of a Critical-Size Defect. Plastic and reconstructive surgery. 2010 Jun;125(6):1685-92.

DAHLIN, C.; ALBERIUS, P.; LINDE, A. Osteopromotion for cranioplasty. An experimental study in rats using a membrande technique. J Neurosurg. v. 74, p. 487-491, 1991.

EINHORN, T.A. Clinically Applied Models of Bone Regeneration in Tissue Engineering Research. Clin. Orthop. Relat. Res. 559-567.1999.

BOYCE et al., 1999; VAN HEESTANDSWINTOWSKI, 1999.

Araujo MG, Lindhe J. Dimensional ridge alterations follwing tooth extraction. An experimental study in the doh. J Clin Periodontol 2005; 32: 212-218.

Araujo MG, Carmagnola D, Berglundh T, Thilander B, Lindhe J. Orthodontic movement in bone defect augmented with Bio-Oss. An experimental study im dogs. J Clin Periodontol. 2001; 28(1):7380 .

Muschleret al., 1996; Lewndrowski et al.,2000; Giannoudis et al.,2005.

AALAMI, OO, NACAMULLI, RP, LENTON, KA, COWAN, CM, FANG, TD, FONG, KD, et al. Applications of a Mouse Model of Calvarial Healing: Differences in Regenerative Abilities of Juveniles and Adults. Plastic and reconstructive surgery. 2004 Sep 1;114(3):713-20.

ABOELSAAD, NS, SOORY, M, GADALLA, LM, RAGAB, LI, DUNNE, S, ZALATA, KR, et al. Effect of Soft Laser and Bioactive Glass on Bone Regeneration in the Treatment of Infra-Bony Defects (a Clinical Study). Lasers in medical science. 2009 May;24(3):387-95

Araujo MG, Carmagnola D, Berglundh T, Thilander B, Lindhe J. Orthodontic movement in bone defect augmented with Bio-Oss. An experimental study im dogs. J Clin Periodontol. 2001; 28(1):7380 .

Barboza E, Oliveira de Souza R, Caula a, Neto LG, Caula F, Duarte ME. Bone regeneration of localized chronic alveolar defects utilizing cell binding peptide associated with ABD bone mineral. $\mathrm{J}$ periodontol 2002; 73: 1153-1159.

Barushka O, Yaakobi T, Oron U. Effect of low-energy laser ( He-Ne) irradiation on the process of bone repair in the rat tibia. Bone. 1995; 16(1): 47-55.

Basfond JR. Low instensity laser therapy: still not an established clinical tool. Lasers Surg Med tissues to osteogenesis. Surg Gynecol obstet 1961; 112:145152.

Basset CA, Creighton DK, Stinchfield FE. Contributions of endosteum, córtex and soft tissues to osteogenesis. Surg Gynecol Obstet 1961; 112:145-152.

Basset CA. Enviromental and cellular factors regulating osteogenesis. Bone Biodinamics 1966; 233244. Beltrán V, Engelke W, Dias FJ, leiva C, Fuebts R, Borie E. Ocllusive in combination with 
particulate Bio-Oss® graft: a pilot study on rabbit calvaria Int J Clin Exp Med. 2014; 15(7):17141720.

Benedicenti A. Manuale di Laser-Terapia del Cavo Orale. Ed. Maggioli. Rimini, 1982.

Chiapasco M, Zaniboni M, Clinical outcomes of GBR procedures to conear periimplant dehistcences a and fenestracions e systematic re view. CI in Oral Implants Res. 2009; 20(4):113-123.

Cho MI, Lin WL, Genco RJ. Plateiet-derived growth fractor-modulated guided tissue regeractive the rapy. J periodontol. 1995; 66: 522-530.

Colangelo P.Piacelli A. Banucci S. Trise P. Formisano G, caiazza S Bone regenacion guided by resobable collagen membranes in rabbiets. Pilot andy. Implant Dent 1993; 2: 101-105.

Colangelo P.Piacelli A. Banucci S. Trise P. Formisano G, caiazza S Bone regenacion guided by resobable collagen membranes in rabbiets. Pilot andy. Implant Dent 1993; 2: 101-105.

Cardoso L, Amade DS. Cardoso M. Clinical results of alveolar redge augmentacion with mandibular biock bone grafts in paet ially priot to implant placement Clin Oral Implant Res. 2002; 13: 103-111. Cunha MJ, Fisper LA, Sbrana MC, de Oliveira PG, do Valle AL, de Almeira AI. Feffect of loe-leve I laser on bone defcts the red wisth in vivo study in calvaria Biomed Res Ine 2014(2014): 1, (ID 104230). Cupher T, Grossman J, Biological of bone graft healing J Foot anide Surg 1996; 35: 413417.

Dahlin C. Alberius P. Linde A. Oste Opromotion for cranioplasy. An ; experimental study in using a menbrane J Neurosurg 19991 b; 74: 487-491.

Boyce et al., 1999 - science.sciencemag.org ;VanHeest and Swintowki, 1999.

Muschler et al.,1996; Lewndrowski et al., 2000; Giannoudis et al., 2005.

Einhom 1995.Mardas et al., 2008; Brug et al., 2000.Schoropp et al., 2003 Araújo et al., 2005;

Araújo et al., 2006. Pietrokovski \& massler 1967; Pietrokovski et al., 2007

Isaksson, 1992, Bergludh \& Lindhe, 1997; Araújo et al., 2011 Trombelli et al.,2002;Esposito et al.,2006; Mards et al., 2010; Badini et al.,2011;

Silvestri et al., 2011;

Jambhekar et al.,2015. Khadra et al., 2004; Merli et al., 2005; Pereira et al.,2009.

Martinez et al., 2008.

Dortbudak et al.,2000; Pinheiro et al.,2003; Stein et al.,2005. Gerbi et al., 2005; Stein et al., 2005; de Almeida et al., 2014;

Cunha et al., 2014. Lugar et al., 1998; Anneroth et al., 1998. Dahlin C Andersson L \& LINDE a. Bone augmentation ae fenstraned implants by an oste operamotive membrane technique A. Clinical Study. Clin Oral Impl Res 1991; 2: 159-169.

Greenwald JA, Mehnn BJ, Spector JA. Fagenhoiz PJ, Saadeh PB, Steinbrech DS. Et al Immanue Versus Mature Dura Mater:II. Differential Expression af gens Important no Calvarial

Reossificaccion. Plast Reconstr Surg 200; 106(3): 630638 discurssion 679.

Gross AJ, Jelkmann W( 1990) Aelium- necon lases irradiaciom inhabits the growth of acidney epithelial Cells inculnere Lasers Surg Red 1040-41

. Hallman M \& Thor A. Bome subtituites and growth factors as an alternavivelcomolement to autogenus bone for grafting in implant dentistry. Periodontol 2000, 2008; 47:172-192. 